\title{
EFICIENCIA DE ENCAPSULACIÓN Y \\ CAPACIDAD DE CARGA DE ANTOCIANINAS DE Vaccinium floribundim Kunt EN NANOPARTICULAS DE ZEINA
}

\section{ENCAPSULATION EFFICIENCY AND LOAD CAPACITY OF ANTHOCYANINS OF Vaccinium floribundim Kunt IN ZEIN NANOPARTICLE}

\author{
Martha Suárez H. ${ }^{*}$ \& Carlos Brito D. ${ }^{1}$ \\ Recibido: 19 de marzo 2019 / Aceptado: 18 de septiembre 2019 \\ DOI: 10.26807/ia.v8i1.98 \\ Palabras clave: antocianinas, zeína, nanopartículas, \\ eficiencia de encapsulación, capacidad de carga \\ Keywords: anthocyanins, zein, nanoparticles, encapsulation efficiency, \\ load capacity
}

\section{RESUMEN}

La zeína es una proteína no polar que tiene un elevado contenido de prolina y leucina, se puede obtener fácilmente de los granos de maíz y por su composición puede formar una matriz proteica de nanopartículas para encapsular colorantes polares. A partir de zeína USP decolorada, se sintetizaron nanopar-

1 Universidad Central del Ecuador, Facultad de Ciencias Químicas, Carrera de Química, Quito- Ecuador (*correspondencia: masuarez@uce.edu.ec) 
tículas por el método de dispersión líquido-líquido. La síntesis se llevó a cabo en etanol al $20 \%(\mathrm{v} / \mathrm{v})$ y a $\mathrm{pH} 4$, condiciones que permiten la más alta estabilidad del sistema. Se obtuvo una suspensión homogénea con un tamaño de partícula de 303,80 nm y una polidispersión de 0,159 que presenta un área superficial de 18,82 $\mathrm{m}^{2} / \mathrm{g}$, se evidenció que las partículas tienen forma esférica y no presentaron porosidad. Las nanopartículas así obtenidas se utilizaron para encapsular antocianinas extraídas del fruto de mortiño (Vaccinium floribundum Kunth) utilizando los métodos de adsorción e incorporación. Se determinó que

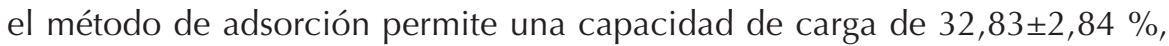

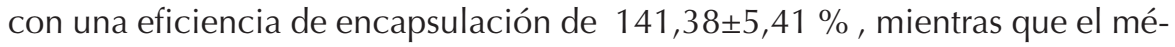

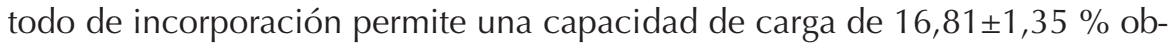

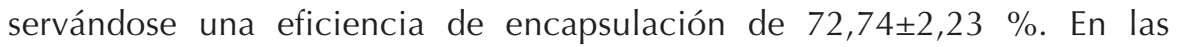
condiciones de estudio los dos métodos producen resultados distintos, siendo más eficiente el método de adsorción.

\section{ABSTRACT}

Zein, a non-polar protein, which has a high proline and leucine content, can be easily obtained from corn grains. Because its composition, can form a protein matrix of nanoparticles to encapsulate polar dyes. From bleached USP, zein nanoparticles were synthesized by the liquid-liquid dispersion method. The synthesis was carried out in $20 \%$ ethanol ( $/ \mathrm{v})$ and at $\mathrm{pH} 4$, conditions that allow the highest system stability. A homogeneous suspension with a particle size of $303.80 \mathrm{~nm}$ and a polydispersion of 0.159 having a surface area of $18.82 \mathrm{~m}^{2} / \mathrm{g}$ was obtained, it was shown that the particles are spherical in shape and have no porosity. The nanoparticles thus obtained were used to encapsulate anthocyanins extracted from the dead fruit (Vaccinium floribundum Kunth) using adsorption and incorporation methods. It was determined that the adsorption method allows a load capacity of $32.83 \pm 2.84 \%$, with an encapsulation efficiency of $141.38 \pm 5.41 \%$, while the incorporation method allows a load capacity of $16.81 \pm 1.35 \%$ with an encapsulation efficiency of $72.74 \pm 2.23 \%$. Under the study conditions, the two methods produce different results, the adsorption method being more efficient. 


\section{INTRODUCCION}

Los sistemas nanoparticulados son coloides denominados "soles", formados por partículas con un tamaño entre $1 \mathrm{~nm}$ y $1000 \mathrm{~nm}$. Su tamaño cercano al de átomos y moléculas, les otorga propiedades únicas tanto a las nanopartículas como a sus suspensiones (Mohanraj \& Chen, 2006). Las nanopartículas empleadas en sistemas de encapsulación se clasifican en nanoesferas y nanocápsulas. Las nanocápsulas son sistemas de membranas poliméricas huecas, que tienen la capacidad de incluir en su cavidad a cualquier molécula, siempre y cuando su tamaño sea menor a la del núcleo de la cápsula (Ersus \& Yurdagel, 2007). En cambio, las nanoesferas son matrices de superficie generalmente porosa, donde las moléculas de interés se encuentran adsorbidas o incrustadas en la superficie de la partícula (Patel \& Velikov, 2011).

Aunque el tamaño de partícula no tiene límites definidos, se consideran nanopartículas cuando éstas tienen un tamaño entre $5 \mathrm{~nm}$ y $1 \mu \mathrm{m}$ (Chang, 2007). El área superficial total del sistema, aumenta conforme disminuye el tamaño de las partículas. Siendo evidente que para estos sistemas los fenómenos y procesos superficiales adquieren gran importancia y de ellos se derivan sus potenciales aplicaciones en el área de la física, química, medicina e ingeniería (Hiemenz \& Rajagopalan, 2017).

Los sistemas nanoparticulados se caracterizan fundamentalmente con el índice de polidispersión y el potencial zeta. El índice de polidispersión es la medida de la distribución de los tamaños de las partículas presentes en una suspensión; se considera que las partículas tienen un tamaño homogéneo cuando la suspensión presenta una polidispersión menor o igual a 0,2 (Bosquez-Molina et al., 2003). El potencial zeta proporciona información sobre la magnitud de las interacciones repulsivas que existen entre las partículas y es una medida de estabilidad de las suspensiones. Además, como es función del entorno iónico de las partículas, se lo puede alterar mediante variaciones del $\mathrm{pH}$ o añadiendo electrolitos a la suspensión (Sandoval et al., 2000). 
Cuando el potencial zeta tiende a cero, las partículas, carentes de carga, se agrupan y la suspensión se desestabiliza. Las nanopartículas constituidas por proteínas presentan un potencial zeta positivo cuando el $\mathrm{pH}$ del sistema es menor a su punto isoeléctrico y cambian a negativo cuando el $\mathrm{pH}$ aumenta sobre su punto isoeléctrico; en el punto isoeléctrico las partículas tienen un potencial zeta cero o cercano a cero (Jones et al., 2010).

En la industria alimenticia, las nanopartículas se utilizan con el fin de desarrollar encapsulantes para colorantes, micronutrientes, nutracéuticos y demás moléculas que requieran ser estabilizadas, para poder ser incorporadas dentro de la formulación de los productos. La carga de compuestos en nanopartículas, que además de estabilizar a las moléculas, es capaz de modificar otras propiedades como su solubilidad, mejorar su biodisponibilidad, permitir una liberación controlada y direccionada de compuestos activos (Bazo et al., 2011; Ezhilarasi et al., 2013; Mohanraj \& Chen, 2006), tiene la ventaja de que el compuesto puede encapsularse en proporciones relativamente altas. Los métodos de encapsulación deben promover una alta capacidad encapsulante para, de este modo, reducir al mínimo la cantidad de la matriz polimérica utilizada (Mohanraj \& Chen, 2006).

La carga de compuestos en nanopartículas se puede realizar por dos métodos: el método de adsorción y el método de incorporación. Para la adsorción de compuestos, las nanopartículas deben ser preparadas y acondicionadas previamente, para eliminar contaminantes superficiales. Los factores que afectan al proceso son: el tipo de adsorción, la velocidad de agitación, la concentración y naturaleza del principio activo, la concentración y propiedades superficiales de las nanopartículas. La incorporación se produce en el momento de la formación de las nanopartículas, el compuesto a encapsular debe estar disuelto en la fase dispersante, la mayor parte es rodeado por el material de la matriz polimérica, formando cápsulas y otra parte es adsorbida en la superficie de las cápsulas formadas. Los factores que influyen en este método son: la velocidad de agitación, la concentración del principio activo y la concen- 
tración de la matriz polimérica (Mohanraj \& Chen, 2006). La eficiencia, de ambos métodos, depende de las interacciones que existen entre el principio activo y la matriz polimérica, además de otros factores como: temperatura, presencia de electrolitos, solubilidad del principio activo, entre otros. La eficiencia de encapsulación (EE) define la cantidad del compuesto que fue cargado, relacionada con la concentración inicial utilizada. La capacidad de carga (LC) por su parte, define la cantidad de compuesto cargado por cada 100 gramos de nanopartículas (Hosseini et al., 2013). Es de esperarse que las nanopartículas con un alto porcentaje de LC y EE sean utilizadas con mayor frecuencia como encapsulantes de compuestos activos.
La zeína es una proteína rica en aminoácidos como la prolina y el ácido glutámico, pero deficiente de aminoácidos esenciales como el triptófano y la lisina, por lo que por sí sola no puede ser considerada como un complemento nutricional (Lawton, 2002). Pero debido a sus propiedades fisicoquímicas, puede ser utilizada para la síntesis de estructuras que permitan la incorporación de compuestos activos, como los colorantes antociánicos, que otorgan propiedades fucionales a los alimentos. El fruto de mortiño (Vaccinium floribundum Kunth) es una fuente muy rica de derivados de la cianidina (Suárez et al., 2018), que son colorantes con características estructurales que permiten su encapsulación en nanopartículas de zeína.

\section{MATERIALES Y METODOS}

Se decoloró zeína grado USP SigmaAldrich CAS: 9010-66-6 de acuerdo al procedimiento sugerido por Suárez \& Mora (2016). La pérdida de color se evalúo mediante un barrido espectrofotométrico entre las longitudes de onda de 400 y $800 \mathrm{~nm}$ en un equipo UV-VIS (CARY50-Bio).
Las antocianinas extraídas del fruto de mortiño (Vaccinium floribundum Kunth) fueron proporcionadas por el Laboratorio de Productos Naturales de la Facultad de Ciencias Químicas de la Universidad Central del Ecuador. 
Síntesis de nanopartículas de zeína por el método de dispersión líquidolíquido

Las condiciones de la síntesis se definieron mediante un diseño factorial completo $2^{2}$ al $95 \%$ de confianza. Los factores de estudio fueron la concentración del medio de dispersión de $10 \%(\mathrm{v} / \mathrm{v})$ y $20 \%(\mathrm{v} / \mathrm{v})$ a dos $\mathrm{pH}$ 4 y 8 . Las variables respuesta fueron el tamaño de partícula, el potencial zeta y la polidispersión.

La síntesis de nanopartículas se basó en el proceso sugerido por Zhong \& Jin (2009). En $30 \mathrm{~mL}$ del medio de dispersión, se agregó $10 \mathrm{~mL}$ de solución al $25 \%$ p/v de zeína, manteniendo un flujo de $1 \mathrm{~mL}$ por minuto a 1600 rpm. Las suspensiones se centrifugaron por 15 minutos a $7500 \mathrm{rpm}$ en una centrífuga de alta revolución (MRC, HSCEN-204). Se aisló el sobrenadante del producto centrifugado.

\section{Caracterización de nanopartículas Potencial zeta}

El potencial zeta se determinó en un equipo de Dispersión Dinámica de Luz (Nano particle, DLS, SZ-100). Mediante una jeringa de $5 \mathrm{~mL}$, se colocó una muestra de cada suspen- sión, dentro de la celda de electroforesis; la celda se colocó en el porta muestras y el equipo se programó en modo zeta potential analyzer.

\section{Índice de polidispersión y tamaño de partícula}

El tamaño de partícula y el índice de polidispersión se determinaron de forma simultánea en el equipo de Dispersión Dinámica de Luz (Nano particle, DLS, SZ-100). Se determinó el índice de refracción de las suspensiones de nanopartículas y se colocó, cada una de las muestras, en una celda de cuarzo de dos ventanas; se programó el software en modo particle size y se procedió a realizar las lecturas.

\section{Características superficiales y área superficial específica}

Se preparó $50 \mathrm{~mL}$ de suspensión de nanopartículas de zeína en una mezcla hidroalcohólica al 20 \% (v/v) y se ajustó el pH a 4 con hidróxido de sodio 0,1 N. Se diluyó $1 \mathrm{~mL}$ de la suspensión de nanopartículas en 10 $\mathrm{mL}$ de etanol (20\%), de esta solución se tomaron $50 \mu \mathrm{L}$ y se goteó sobre un cubre objetos previamente calentado a $90{ }^{\circ} \mathrm{C}$. El análisis superficial se rea- 
lizó después de 72 horas de secado, en modo de no contacto en un Microscopio de Fuerza Atómica (Park Systems, NX10). El área superficial específica fue calculada a partir de la distribución del tamaño de partícula.

\section{Encapsulación de antocianinas}

Para definir la metodología que permita maximizar la carga de antocianinas en nanopartículas de zeína, se realizó un estudio comparativo entre los métodos de adsorción e incorporación, para lo cual se empleó un diseño estadístico unifactorial, al 95\% de confianza. El factor de estudio fue el tipo de método y las variables respuesta, la capacidad de carga (\%LC) y la eficiencia de encapsulación (\%EE).

\section{Encapsulación de antocianinas por el método de adsorción}

La suspensión de nanopartículas se congeló en un ultra congelador-80 (Arctiko, ULUF 450) y se deshidrató en un liofilizador (Telstar, Lyoalfa 10/85). Se preparó una solución de antocianinas $0,5 \%(\mathrm{~m} / \mathrm{v})$ en etanol $20 \%$ y se agregaron las nanopartículas de zeína liofilizadas hasta una concentración final de $1 \mathrm{mg} / \mathrm{mL}$ de nanopartículas. La carga se llevó a cabo mediante agitación magnética a 1600 rpm por 30 minutos.

\section{Encapsulación de antocianinas por el método de incorporación}

Se agregó a la solución etanólica de antocianinas de concentración 0,5 \% $(\mathrm{m} / \mathrm{v})$ la solución hidroalcohólica de zeína decolorada 1,6 \% (m/v). La incorporación se realizó utilizando la misma metodología descrita para la síntesis de nanopartículas.

\section{Determinación de la eficiencia de encapsulación y de la capacidad de carga}

La cuantificación de cianidina-3-glucósido antes y después de la carga en nanopartículas se realizó en un espectrofotómetro UV-VIS (VARIAN BioCary 50) a $520 \mathrm{~nm}$. Se elaboró una curva de calibración con un estándar primario de cianidina-3-glucósido (Sigma Aldrich CAS: 7084-24-4) utilizando como solvente una mezcla hidroalcohólica 80:20 a pH 4. Se utilizó un extracto etanólico seco de antocianinas con una concentración de cianidida 3-glucósido de 3,9 \pm 0,4 $\mathrm{mg} / \mathrm{g}$ de extracto seco, sin ningún tratamiento previo. 


\section{RESULTADOS}

Síntesis y caracterización de nanopartículas

En las dos concentraciones de la fase dispersante las suspensiones fueron visiblemente homogéneas, con una polidispersión menor que 0,2 pará- metro que de acuerdo a BosquezMolina et al. (2003), asegura homogeneidad en el tamaño. Sin embargo, a $\mathrm{pH}$ 4, se observa que los tamaños de partícula disminuyen (Tabla 1).

Tabla 1. Tamaño de partícula, polidispersión y potencial zeta de las nanopartículas de zeína, sintetizadas en los diferentes medios de dispersión

\begin{tabular}{cccccc}
\hline $\mathbf{N}^{\circ}$ & $\begin{array}{c}\text { Medio de } \\
\text { dispersión } \\
\text { (\%etanol) }\end{array}$ & pH & $\begin{array}{c}\text { Tamaño } \\
\text { partícula } \\
(\mathrm{nm})\end{array}$ & Polidispersión & $\begin{array}{c}\text { Potencial } \\
\text { zeta } \\
(\mathrm{mV})\end{array}$ \\
\hline 1 & 10 & 4 & 282,05 & 0,127 & $+13,2$ \\
2 & 20 & 4 & 303,80 & 0,159 & $+18,9$ \\
3 & 10 & 8 & 346,85 & 0,189 & $-14,4$ \\
4 & 20 & 8 & 359,50 & 0,177 & $-12,25$ \\
\hline
\end{tabular}

El valor de potencial zeta para las nanopartículas varió de acuerdo a las condiciones de síntesis, observándose que a $\mathrm{pH} 4$ y $20 \%$ de etanol, las nanopartículas presentaron mayor estabilidad con una carga superficial de $+18,9 \mathrm{mV}$, que concuerda con la definición de Díaz et al., (2016) que consideran que los valores de potencial zeta mayores que $+30 \mathrm{mV}$ y menores que $-30 \mathrm{mV}$ promueven una alta estabilidad y evitan que las par- tículas lleguen al fenómeno de agregación. Sin embargo, de acuerdo a los valores presentados en la Tabla 1 , estas partículas presentan un valor de polidispersión más alto, teniéndose un sistema menos homogéneo.

La disminución observada en el tamaño de partícula se explica por la relación inversa entre éste y la concentración de zeína (Zhong \& Jin, 2009). 


\section{Determinación de las características superficiales}

Para el análisis de las características superficiales, se utilizó la suspensión de nanopartículas de zeína, las cuales presentaron un tamaño de partícula de 303,8 nm y una polidispersión de 0,159. La distribución obtenida mediante DLS mostró que el tamaño de las partículas obtenidas varía entre 100 y $350 \mathrm{~nm}$, que correspondió a un rango de polidispersión menor comparado con el obtenido por Bazo et al. (2011), que se ubicó entre 100 y $500 \mathrm{~nm}$ para una suspensión de partículas de aproximadamente 200 nm de tamaño.

Las imágenes de las nanopartículas de zeína, tomadas con el microscopio de fuerza atómica se presentan en la Figura 1. Se puede observar que las partículas tienen forma esférica, no se pudo evidenciar la presencia de poros en la superficie de las mismas. Las partículas presentaron un área superficial específica de 18,82 $\mathrm{m}^{2} / \mathrm{g}$.

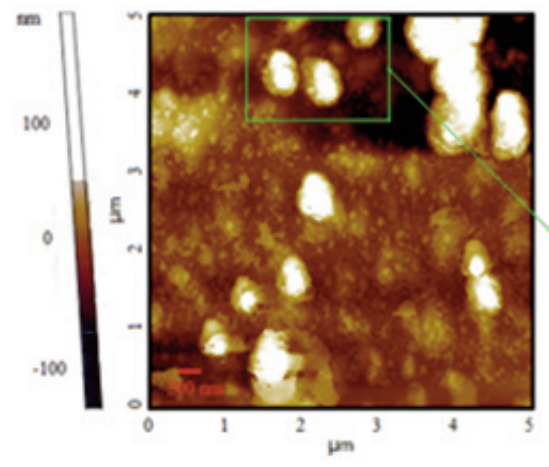

a)

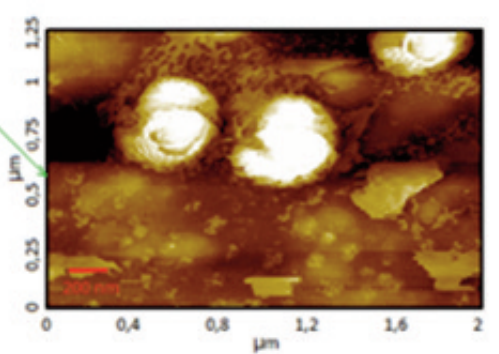

b)

Figura 1. Imágenes de microscopio de fuerza atómica (AFM) de nanopartículas de zeína. (a) Área de análisis de 5 × $5 \mu \mathrm{m}$ y (b) Acercamiento de 1,25 × $2 \mu \mathrm{m}$

\section{Encapsulación de antocianinas}

Se utilizaron nanopartículas desarro- lladas a partir de una solución de zeína de concentración $16 \%(\mathrm{~m} / \mathrm{v})$. En estas condiciones se evaluaron los 
procesos de encapsulación y se cuantificó la cianidina-3-glucósido mediante el método espectrofotométrico. La solución de antocianinas extraídas del fruto del mortiño (Vaccinium floribundum Kunth), presentó una longitud de onda máxima de 520 $\mathrm{nm}$, debido a la presencia de cianidina-3-glucósido. El resultado concuerda con el valor reportado por Suárez \& Narváez, (2016) de 515,0 $\mathrm{nm}$ en presencia de solución buffer de $\mathrm{pH}$ 4. La ecuación de la curva de calibración, obtenida a partir de ocho patrones de cianidina-3-glucósido, fue: $A=0,0365 C-0,0315$, con un coeficiente de correlación $\left(\mathrm{R}^{2}\right)$ de 0,9981, la concentración se evaluó en $\mu \mathrm{g} / \mathrm{mL}$.

Los resultados de eficiencia de encapsulación y capacidad de carga de los métodos de adsorción e incorporación, se muestran en la Tabla 2 .

A partir de los datos obtenidos se realizó el análisis ANOVA, con un análisis de varianza para cada respuesta, el mismo que establece que los métodos de adsorción e incorporación provocan resultados distintos.

\section{Tabla 2. Eficiencia de encapsulación (\%EE) y capacidad de carga (\%LC) de cianidina-3-glucósido en nanopartículas de zeína por los métodos de adsorción e incorporación}

\begin{tabular}{cccc}
\hline \multicolumn{2}{c}{$\begin{array}{c}\text { Método de adsorción } \\
\text { (\%EE) }\end{array}$} & (\%LC) & \multicolumn{2}{c}{$\begin{array}{c}\text { Método de incorporación } \\
\text { (\%EE) }\end{array}$} & (\%LC) \\
\hline $32,83 \pm 2,84$ & $141,38 \pm 5,41$ & $16,81 \pm 1,35$ & $72,74 \pm 2,23$ \\
\hline
\end{tabular}

Los resultados son reportados como media $\pm S D, n=11$

\section{DISCUSION}

Síntesis de nanopartículas por el método de dispersión líquido- líquido

De acuerdo a los datos de la Tabla 1, los medios de dispersión 3 y 4 ajustados a pH 8, presentan partículas con carga superficial negativa, esto puede deberse a que en medio alcalino la proteína presenta los grupos amonio y carboxilo desprotonados (Patel et al. 2010), de acuerdo al equilibrio de reacción que se muestra en la Figura 2. 


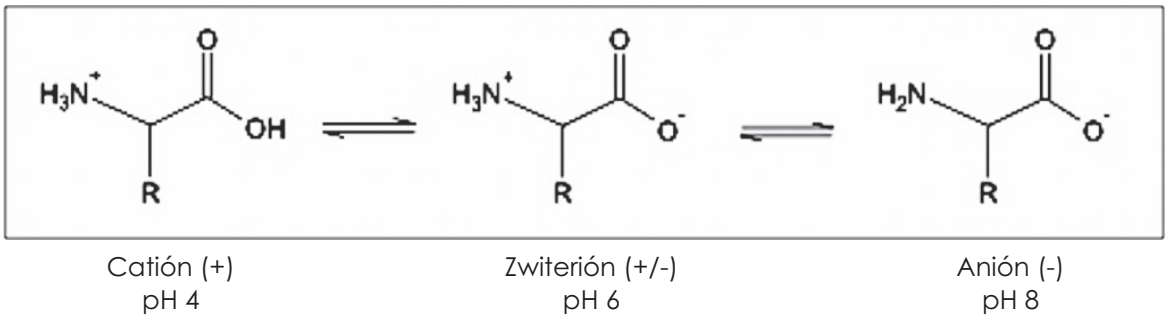

Figura 2. Representación de las estructuras químicas predominantes de zeína, a diferentes valores de pH. Modificado de Blanco et al., (2013)

La zeína presenta su punto isoeléctrico en $\mathrm{pH}$ 6, en el cual predomina la estructura química del zwitterión, donde la proteína tiene sus grupos funcionales amino y carboxilo ionizados, con carga total neutra. $\mathrm{A} \mathrm{pH} 4$, ambos grupos se encuentran protonados, por lo que predomina la forma catiónica y a $\mathrm{pH} 8$ se muestra favorecida la forma aniónica (Shukla \& Cheryan, 2001), lo que explica el signo obtenido para el potencial zeta.

El análisis estadístico para el diseño factorial completo $2^{2}$ al $95 \%$ de confianza, de los datos cuyas medias se presentan en la Tabla 1, demuestra que a pesar de que el efecto de la concentración de etanol no es significativo en zeta, éste se ve significativamente afectado por el $\mathrm{pH}$ y la interacción de los efectos de los factores, concentración de etanol y $\mathrm{pH}$.
El cambio de $\mathrm{pH}$ de 4 a 8 , provoca un decremento en el potencial zeta, lo que tiene como consecuencia la disminución de la estabilidad de la suspensión. El efecto de la interacción de los factores concentración de etanol y $\mathrm{pH}$, puede deberse a que la molécula de etanol compite con la proteína en las reacciones de neutralización ocasionadas por los iones hidronio $\left(\mathrm{H}_{3} \mathrm{O}^{+}\right)$e hidroxilo $(-\mathrm{OH})$ de los medios ácidos y básicos utilizados (Zhong \& Jin, 2009). De acuerdo a Patel et al. (2010), el valor máximo del potencial zeta no debe superar los $\pm 31 \mathrm{mV}$, para considerarse como una suspensión moderadamente estable y con un valor mínimo de $\pm 10,3 \mathrm{mV}$ se tienen suspensiones con ligera estabilidad, experimentalmente las nanopartículas más estables se obtuvieron en etanol $20 \%$ y $\mathrm{pH} 4$, con un potencial zeta de $+18,9$ 
$\mathrm{mV}$, que concuerda con las observaciones de Patel et al. (2010). La estabilidad está influenciada por la concentración de etanol, debido a que la parte hidrófoba de la molécula interacciona con la superficie de la partícula de zeína y la sección hidrófila se proyecta hacia el seno de la solución, formando una capa protectora y ac-

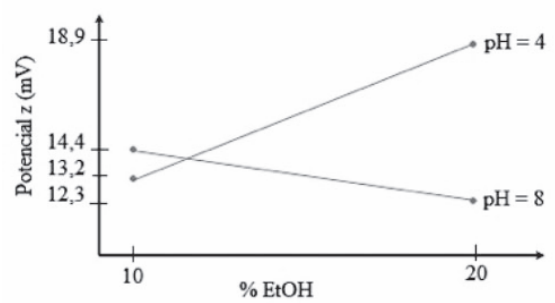

Figura 3. Interacción de los factores \%EłOH y pH en el potencial zeta

En el estudio de los efectos provocados sobre el tamaño de partícula, se encontró que la concentración de etanol y la interacción de los factores no causan un efecto significativo, por lo que el efecto del $\mathrm{pH}$ puede ser analizado independientemente. Este resultado puede ser explicado por la corta distancia entre los niveles mínimos y máximos de la concentración de etanol. El cambio en el $\mathrm{pH}$ de $4 \mathrm{a}$ 8 provoca un incremento en el tamaño de las nanopartículas, pero debido a que en el desarrollo de nano-

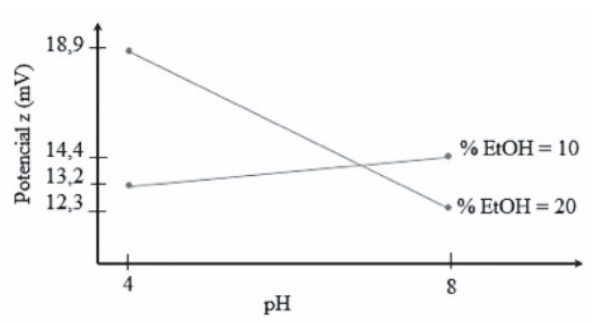

tuando como tensoactivo, fenómeno que de acuerdo a los datos obtenidos está influenciado por el $\mathrm{pH}$. En la Figura 3, se puede observar que la interacción es de carácter inverso, lo que significa que el efecto de la concentración de etanol depende del valor del $\mathrm{pH}$.

estructuras se busca que las mismas alcancen el menor tamaño posible, el incremento en el tamaño resulta perjudicial (Hosseini et al., 2013).

La disminución observada en el tamaño de partícula también se puede explicar, por la relación inversa entre éste y la concentración de zeína (Zhong \& Jin, 2009).

En cambio, para el caso de la polidispersión se encontró que ningún efecto es significativo, la polidispersión 
depende en mayor medida de los métodos para la síntesis y purificación de las nanopartículas (Bazo et al., 2011).

\section{Eficiencia de encapsulación y capa- cidad de carga}

Los resultados presentados en la Tabla 2 demostraron que el proceso de carga de antocianinas en nanopartículas de zeína, es de carácter superficial. La cantidad de cianidina-3glucósido retenida en la superficie adsorción-, es mayor a la cantidad encapsulada en el interior de la partícula-incorporación-. En el método de incorporación las nanopartículas se forman dentro de la solución de antocianina, por lo que las moléculas de etanol del medio de dispersión, rodean inmediatamente a la partícula, compitiendo con las antocianinas en los procesos de adsorción y de esta manera saturan inmediatamente la superficie de las mismas, datos que son coincidentes con la investigación de Luo et al., (2012).

\section{CONCLUSIONES}

La más alta estabilidad de las nanopartículas de zeína se da cuando el medio dispersante tiene una concentración de etanol del $20 \%(\mathrm{v} / \mathrm{v})$ y un $\mathrm{pH}$ 4. En estas condiciones se obtuvo una suspensión homogénea de nanopartículas de zeína, con un tamaño de 303,8 nm y una polidispersión de 0,159 . Se definió el método para sintetizar nanopartículas de zeína mediante dispersión líquido-líquido, las cuales se caracterizaron con índice de polidispersión, tamaño de partícula y potencial zeta que se encuentran dentro de los parámetros establecidos teóricamente. En las nanopartículas sintetizadas, se determinó la viabilidad de encapsular antocianinas encontrándose que la eficiencia de encapsulación y la capacidad de carga obtenidas permiten desarrollar colorantes antociánicos encapsulados. 


\section{LISTA DE REFERENCIAS}

Bazo, M. A., Catalán, I. E., Ferrero, C. G., Navarro, C. J. G., Garreta, J. M. I., \& Hualde, A. R. (2011). Nanoparticles for encapsulation of compounds, the production and uses thereof. Google Patents.

Bosquez-Molina, E., Guerrero-Legarreta, I., \& Vernon-Carter, J. E. (2003). Moisture barrier properties and morphology of mesquite gum-candelilla wax based edible emulsion coatings.Food Research International, 36 (9); 885-893.

Chang, R. (2007). Química General, 9a edición, Ed. McGraw-Hill.

Díaz, D., Jiménez, M., \& Lugo, E. (2016). Propiedades Fisicoquímicas y Estabilidad de la Oleorresina de Paprika Micro y Nano Encapsulada. Universidad Veracruzana.

Ersus, S., \& Yurdagel, U. (2007). Microencapsulation of anthocyanin pigments of black carrot (Daucuscarota L.) by spray dryer. Journal of Food Engineering, 80(30), 805812.

Ezjilarasi, P., Chhanwal, N., \& Chinnaswamy C. (2013). Nanoencapsulation Techniques for Food Componenets: A Review. Food Bioprocess Technol.

Hiemenz, P. C., \& Rajagopalan, R. (2017). Principles of Colloid and Surface Chemistry, Third Edition, Revised and Expanded. CRC Press.

Hosseini, S. F., Zandi, M., Rezaei, M., \& Farahmandghavi, F. (2013). Two-step method for encapsulation of oregano essential oil in chitosan nanoparticles: Preparation, characterization and in vitro release study. Carbohydrate Polymers, 95(1), 50-56.

Jones, O., Decker, E. A., \& McClements, D. J. (2010). Thermal analysis of lactoglobulin complexes with pectins or carrageenan for production of stable biopolymer particles. Food Hydrocolloids, 24(2-3), 239-248.

Lawton, J. W. (2002). Zein: A history of processing and use. Cereal Chemistry, 79(1), 118. 
Luo, Y., Teng, Z., \& Wang, Q. (2012). Development of zein nanoparticles coated with carboxymethyl chitosan for encapsulation and controlled release of vitamin D3. Journal of Agricultural and Food Chemistry, 60(3), 836-843.

Mohanraj, V. J., \& Chen, Y. (2006). Nanoparticles - A Review. Tropical Journal of Pharmaceutical Research, 5(1), 561-573.

Patel, A. Bouwens, E., \& Velikov, K., (2010). Sodium caseinate stabilized zein colloidal particles. Journal of agricultural and food chemistry, 58(23), $12497-12503$.

Patel, A. R., \& Velikov, K. P. (2011). Colloidal delivery systems in foods: A general comparison with oral drug delivery. LWT - Food Science and Technology, 44(9), 19581964.

Sandoval, L., Montellano, L., Piña, M. \& Sánchez, L., (2000). Potencial zeta como una herramienta para determinar la aglomeración de las partículas en la reducción del volumen del lodo a disponer. Federación Mexicana de Ingeniería Sanitaria y Ciencias Ambientales; AIDIS. Ciencia y conciencia compromiso nacional con el medio ambiente: memorias técnicas. México, D.F, FEMISCA, 2000. p.1-15.

Shukla, R., \& Cheryan, M. (2001). Zein: The industrial protein from corn. Industrial Crops and Products, 13(3), 171-192.

Suárez, M., \& Narváez, G. (2016). Copigmentación intermolecular de antocianinas glicosiladas (Primera). Quito: Editorial Académica Española.

Suárez, M., \& Mora, S. (2016). Decoloración de zeína. Universidad Central del Ecuador, Laboratorio de Productos Naturales, Quito Ecuador.

Suárez, M., Yazán, E., \& Narváez, G. (2018). Influencia de la estabilización por copigmentación intermolecular sobre la capacidad antioxidante de antocianinas del fruto de Vaccinium floribundum Kunt. Infoanalítica 6(1), 39-53.

Zhong, Q., \& Jin, M. (2009). Zein nanoparticles produced by liquid-liquid dispersion. Food Hydrocolloids, 23(8), 2380-2387. 